Article

\title{
An Automated Hierarchical Approach for Three-Dimensional Segmentation of Single Trees Using UAV LiDAR Data
}

\author{
Wanqian Yan ${ }^{1}$, Haiyan Guan ${ }^{2, *}$, Lin Cao ${ }^{3 \oplus}$, Yongtao $\mathrm{Yu}^{4}$, Sha Gao ${ }^{3}$ and Jian Yong $\mathrm{Lu}^{5}$ \\ 1 School of Geographical Science, Nanjing University of Information Science \& Technology, \\ Nanjing 210044, China; ywq_nuist@163.com \\ 2 School of Remote Sensing \& Geomatics Engineering, Nanjing University of Information Science \\ \& Technology, Nanjing 210044, China \\ 3 Co-Innovation Center for Sustainable Forestry in Southern China, Nanjing Forestry University, \\ 159 Longpan road, Nanjing 210037, China; lincao@njfu.edu.cn (L.C.); nfu_gaosha@126.com (S.G.) \\ 4 Faculty of Computer and Software Engineering, Huaiyin Institute of Technology, Huai'an 223003, China; \\ allennessy.yu@gmail.com \\ 5 Institute of Space Weather, School of Math \& Statistics, Nanjing University of Information Science \\ \& Technology, Nanjing 210044, China; jylu@nuist.edu.cn \\ * Correspondence: guanhy.nj@nuist.edu.cn; Tel.: +86-25-5873-1152
}

Received: 5 November 2018; Accepted: 8 December 2018; Published: 10 December 2018 updates

\begin{abstract}
Forests play a key role in terrestrial ecosystems, and the variables extracted from single trees can be used in various fields and applications for evaluating forest production and assessing forest ecosystem services. In this study, we developed an automated hierarchical single-tree segmentation approach based on the high density three-dimensional (3D) Unmanned Aerial Vehicle (UAV) point clouds. First, this approach obtains normalized non-ground UAV points in data preprocessing; then, a voxel-based mean shift algorithm is used to roughly classify the non-ground UAV points into well-detected and under-segmentation clusters. Moreover, potential tree apices for each under-segmentation cluster are obtained with regard to profile shape curves and finally input to the normalized cut segmentation (NCut) algorithm to segment iteratively the under-segmentation cluster into single trees. We evaluated the proposed method using datasets acquired by a Velodyne 16E LiDAR system mounted on a multi-rotor UAV. The results showed that the proposed method achieves the average correctness, completeness, and overall accuracy of $0.90,0.88$, and 0.89 , respectively, in delineating single trees. Comparative analysis demonstrated that our method provided a promising solution to reliable and robust segmentation of single trees from UAV LiDAR data with high point cloud density.
\end{abstract}

Keywords: UAV LiDAR; single tree; segmentation; mean shift; improved normalized cut

\section{Introduction}

Forests play a key role in the terrestrial ecosystem and have a large amount of economic, ecological, and social benefits because they regulate the water cycle and carbon cycle on the surface of the Earth [1]. For effective forest management and human activity documentation, the forest inventory for sustainable forest management is usually needed to obtain a great number of single tree-related parameters, such as tree species and tree species distribution, timber volume, increment of timber volume, and mean tree height [2-9]. Traditionally, in practice, most single-tree-level parameters are estimated by manually sampling small plots in a field survey, which is time consuming and labor intensive [10,11]. 
In recent years, airborne light detection and ranging (LiDAR) has become an emerging remote sensing technique for performing forest inventory tasks because it can penetrate through tree canopies and acquire three-dimensional (3D) forest structures [12,13]. LiDAR technology can improve the accuracy of forest parameter retrieval at the single-tree level because of its capability of providing accurate and spatially detailed information of tree structure elements (such as branches and foliage) [14]. Currently, airborne LiDAR is considered to be a standard data source for deriving forest spectral and spatial information at the scale of single trees because it provides timely, large-scale, and accurate forest information to support forest management. Matasci et al. [2] demonstrated that the integration of airborne LiDAR and Landsat-derived reflectance products predicted a total of 10 forest structural attributes by using a nearest neighbor imputation approach based on the random forest framework, with $R^{2}$ values ranging from $0.49-0.61$ for key response variables such as canopy cover, stand height, basal area, and stem volume. Allouis et al. [3] estimated stem volume and aboveground biomass from the single-tree metrics derived from full-waveform LiDAR data by using regression models and improved the accuracy of aboveground biomass estimates. Matsuki et al. [4] obtained a tree species classification accuracy of $82 \%$ by integrating spectral features obtained from hyperspectral data and tree-crown features derived from LiDAR data with a support vector machine classifier. However, it is still difficult to detect single trees automatically from airborne LiDAR data due to the various shapes of trees and their periodic changes with the seasons, especially to segment trees with complex and heterogeneous crowns.

With the development of unmanned aerial vehicles (UAV) technologies in weight capacity, durability, and controlling, UAV LiDAR systems have been proposed to be an alternative means for capturing 3D canopy structural information. Compared to airborne LiDAR, UAV LiDAR has been shown to be lower cost, which can monitor key development stages of forest management in a timely manner, such as pruning, thinning, and harvesting [15]. Moreover, it is more flexible and controllable in terms of flying altitude, viewing angles, and forward and side overlap with fine spatial and temporal resolutions [16]. Furthermore, UAV LiDAR can avoid some complications, such as plane flight logistics, cloud covers, and atmospheric effects [17]. Because UAV and airborne LiDAR data show certain similar characteristics, most researchers segmented single trees from UAV LiDAR data by using the algorithms that are usually applied to airborne LiDAR data.

Many methods have been proposed for detecting and delineating single trees from airborne LiDAR points. These methods can be generally categorized into two categories, i.e., raster-based and point-based methods, in terms of data types used [18]. Raster-based methods first interpolate point clouds into a two-dimensional (2D) canopy height model (CHM), in which potential stem positions are located based on the local maxima, and then, tree crowns are delineated by using the established image processing algorithms, such as watershed segmentation, valley following, template matching, and region growing [19-21]. However, raster-based methods heavily rely on the accuracy of the constructed CHM. Moreover, these methods usually fail to detect and delineate some smaller trees under the canopies and closely neighboring trees especially in dense and heterogeneous forests [22]. Consequently, some improvements have been proposed. For example, a filtering CHM and variable window strategy have been presented to reduce the misclassification of local maxima caused by the noise in CHM [20,21]. Wallace et al. [15] demonstrated that high density UAV LiDAR points contributed to the improvement of the tree extraction accuracy. Hyyppä et al. [1] detected trees by using the last pulse data, which improved the tree detection accuracy by $6 \%$. However, these raster-based methods use only tree elevation information of the constructed CHM, and 3D spatial information of LiDAR data has been ignored [23].

To make full use of the 3D structures of point clouds, in recent years, many researchers have identified single trees directly from original LiDAR data [23-25]. Morsdorf et al. [26] segmented trees in a 3D voxel space with a $k$-means clustering method by using the local maxima of the CHM as seed points. Gupta et al. [27] improved Morsdorf's method by scaling down height values to increase the similarity between point clouds. However, these methods also relied on the local maxima detected 
in CHM data. Region growing has been widely used in single-tree delineation from airborne LiDAR data. Lee et al. [21] first defined an optimal moving window size to detect all possible seed points with an overall accuracy of $95.1 \%$. However, it required ground truth data as training data to achieve the optimal results. Li et al. [22] proposed a top-to-down region-growing algorithm based on the relative tree spacing to detect single trees. The method performed well in conifer forest, but it worked less effectively when applied to a deciduous forest. Lu et al. [25] segmented single trees with an overall F-score of 0.9. However, the method required intensity information and could be only applied to leaf-off conditions, which limited the further applications. These clustering methods are unsuitable to deal with forests with overlapped canopies because they can hardly find appropriate parameters for large-scale forest environments or staggered stems in deciduous forests. Moreover, the above single-tree segmentation methods have certain restrictions on tree species and LiDAR data, and their segmentation accuracies on overlapped canopies are usually unsatisfactory.

Ferraz et al. [28] presented an improved mean shift algorithm, considering both density and height factors to detect tree apices in multi-layered forests. Dai et al. [29] improved the tree detection accuracy by using multispectral airborne LiDAR. A mean shift method is capable of locating tree apices according to the density and height maxima of point clouds without prior knowledge of the number of clusters or depending on the interpolated 2D CHM data. To delineate small trees under canopies from airborne LiDAR data, Reitberger et al. [30] introduced normalized cut (NCut), originally proposed for $2 \mathrm{D}$ image segmentation, and achieved a recognition rate of $12 \%$, which is higher than conventional watershed segmentation methods. Zhong et al. [31] improved the algorithm to segment overlapped trees with a correctness of above $90 \%$ by means of terrestrial and mobile LiDAR data. NCut has shown great potential in 3D segmentation and works very well when separating two overlapping objects [32,33]. However, the traditional NCut has difficulty in accurately locating tree stems in airborne/UAV LiDAR data due to the lack of understory information in severely-overlapped forests.

The objective of this study aims to propose an automated hierarchical single-tree delineation approach, as well as applying and assessing the feasibility of the proposed algorithm using high-density UAV LiDAR data. The remainder of the paper is organized as follows: Section 2 describes the study area and UAV LiDAR data acquired from a Velodyne 16E system. Section 3 details the proposed single-tree segmentation method using UAV LiDAR data. The method starts with the normalization and segmentation of non-ground points from the Velodyne 16E UAV LiDAR data. After that, to improve data processing efficiency, a voxel-based mean shift algorithm is used to roughly obtain well-detected and under-segmentation clusters. Finally, to delineate overlapped trees effectively, an improved normalized cut (NCut) segmentation method is proposed to segment under-segmentation clusters iteratively into single trees with a tree apex detection strategy. The conducted tests are described and analyzed in Section 4. Finally, concluding remarks are given in Section 5.

\section{Test UAV LiDAR Data}

The study area (marked by a large yellow frame in Figure 1) is about 54.67 hectares and located in Dongtai forest farm with relatively flat terrain, Yancheng City, Jiangsu, China. The specific location of the study area is detailed in Figure 1. The trees in the forest farm were planted in almost the same period of time, leading to approximate similarities of these trees in shape and size. The test site dominantly covers two tree species: Metasequoia and Poplar. Metasequoia, a fast-growing, deciduous tree, and the sole living species, has a cone-shaped canopy with sparse branches and leaves, as shown in Figure 2a. Poplar, a genus of 25-35 species of deciduous flowering plants, is among the most important boreal broadleaf trees in northern cities of China. Poplar has an irregular shape (see Figure 2b) with spirally-arranged leaves that vary in shape from triangular to circular or lobed, and with a long petiole. 


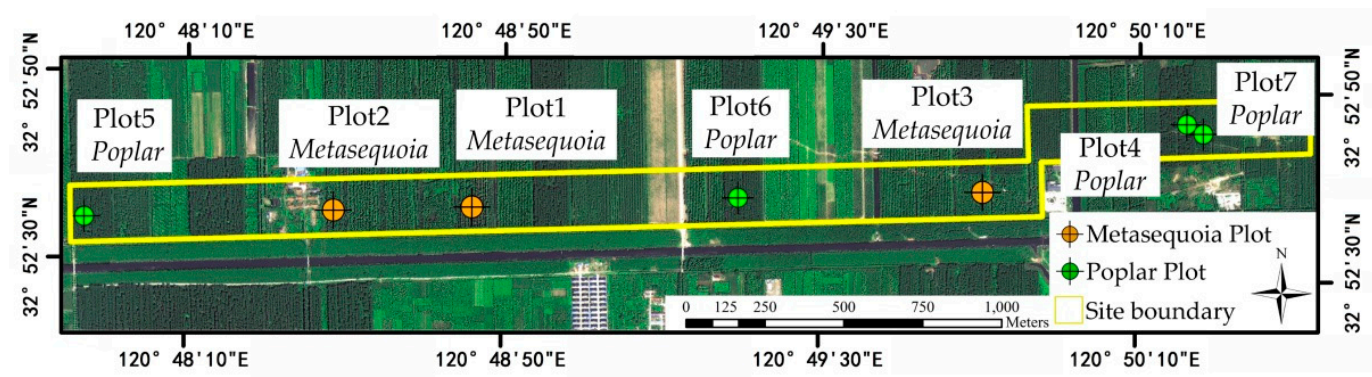

Figure 1. The study area and the spatial distribution of field plots.

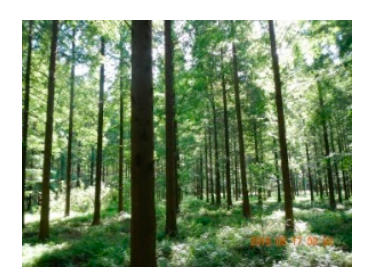

(a)

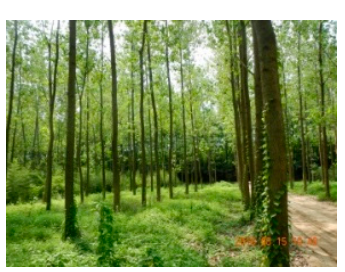

(b)

Figure 2. Two tree species in the study site (a) Metasequoia and (b) Poplar.

The research data were collected by a laser scanner system mounted on a GV1300 multi-rotor UAV produced by Green Valley International. The serial number of the UAV LiDAR system is LA2016-003. The UAV platform is composed of the following components: eight brushless motors, a Novatel initial measurement unit (IMU-IGM-S1), a dual-frequency GPS (global positioning system) produced by Novatel, and a ground control system to track the aircraft, control the UAV-LiDAR system, and continuously monitor UAV flying parameters. The main components of the UAV platform can be seen in Figure 3. Lin et al. [34] demonstrated the advantages of these sensors integrated on a UAV platform and the feasibility of the UAV development. Besides, a Novatel global navigation satellite system ground base station was used to ensure GPS accuracies. The real-time UAV LiDAR data were transferred to the ground data terminals through a long-range Wi-Fi system connected to the UAV [35]. The parameters of the UAV platform are listed in Table 1.

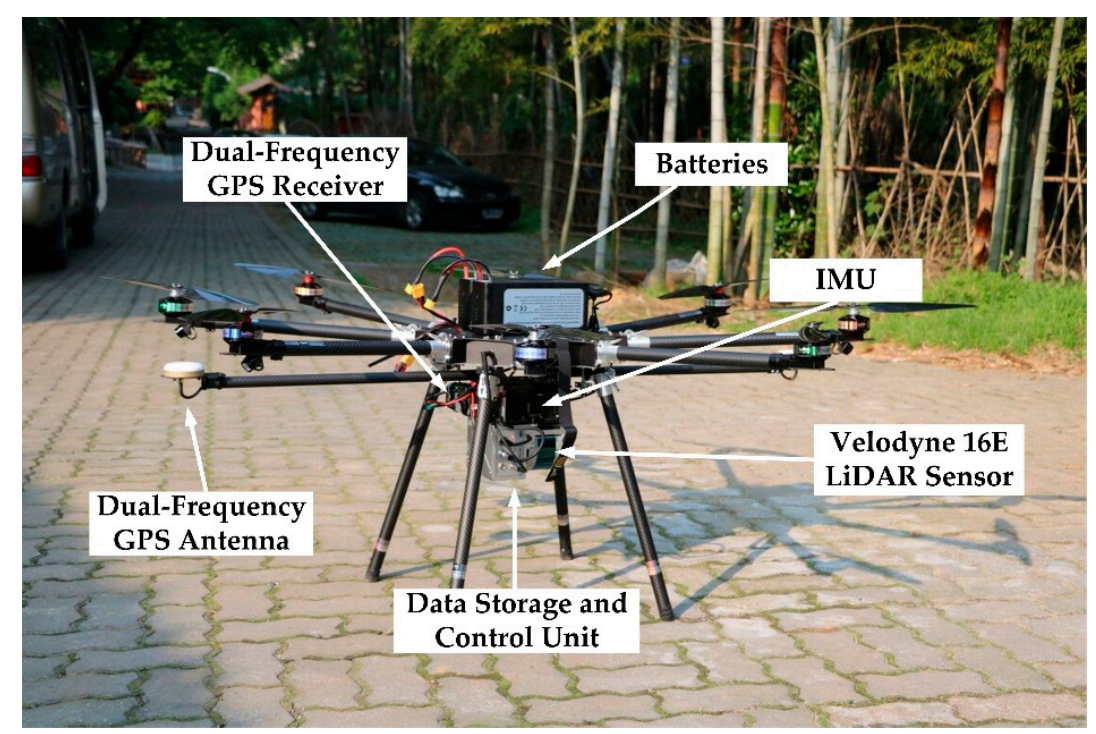

Figure 3. Main components of the UAV platform and Velodyne 16E laser scanner system. 
Table 1. Specifications of the UAV platform.

\begin{tabular}{ll}
\hline Parameters & Value \\
\hline Weight of loadings & $7 \mathrm{~kg}$ \\
Take-off-weight & $17 \mathrm{~kg}$ \\
Diagonal wheelbase & $1280 \mathrm{~mm}$ \\
Maximum flying time & $32 \mathrm{~min}$ \\
Maximum flying distance & $10 \mathrm{~km}$ \\
Flying height & $86 \mathrm{~m}$ above ground in this research \\
Flying speed & $3.6 \mathrm{~m} / \mathrm{s}$ in this research \\
Measurement range of gyroscope & \pm 400 degrees $/ \mathrm{s}$ \\
Gyroscope zero drift & $0.5 \mathrm{degrees} / \mathrm{h}$ \\
Measurement range of accelerometer & $\pm 10 \mathrm{~g}$ \\
Measurement deviation of accelerometer & $0.05 \mathrm{mg}$ \\
\hline
\end{tabular}

The UAV LiDAR data were acquired with a point density of 40.57 points $/ \mathrm{m}^{2}$ on $24-26$ July 2017, by a VLP16 (Velodyne 16E) laser scanner system produced by Velodyne LiDAR. The full specifications of VLP16 are listed in Table 2. Note that, in this study, the LiDAR data accuracy, which usually requires being evaluated based on the ground control points measured by a total station and creating a reference dataset [36,37], was unavailable; therefore, the data accuracy was directly reflected by the system's ranging accuracy.

Table 2. Specifications of the Velodyne 16E laser scanner system.

\begin{tabular}{cc}
\hline Parameters & Value \\
\hline Principle & Pulse ranging \\
Laser wavelength & $905 \mathrm{~nm}$ \\
Measurement distance range & $100 \mathrm{~m}$ \\
Vertical scan angle & $-15-+15 \mathrm{degrees}$ \\
Laser scan frequency & 16 lines $/ \mathrm{s}$ \\
Divergence & $3 \mathrm{mrad}$ \\
Swath overlap & $100 \%$ \\
Amplitude & $500 \mathrm{~m} / \mathrm{s}^{2}$ \\
Vibration frequency & $5-2000 \mathrm{~Hz}, 3 \mathrm{Grms}{ }^{1}$ \\
Weight & $0.83 \mathrm{~kg}$ \\
Ranging accuracy & $<10 \mathrm{~cm}$ \\
\hline
\end{tabular}

\footnotetext{
${ }^{1} \mathrm{Grms}$ is a unit for representing root mean square acceleration.
}

To evaluate the single-tree segmentation accuracy, the reference data were collected in July 2017, by field measurements and a backpack laser scanning system produced by Green Valley International. The full specifications of the backpack laser scanning system are listed in Table 3. According to the producer, the data accuracy was about $5 \mathrm{~cm}$. Seven reference plots were selected to evaluate the single-tree segmentation accuracy in the study site, as shown in Figure 1, including three samples of Metasequoia (Plots 1, 2, and 3) and four samples of Poplar (Plots 4, 5, 6, and 7). Each tree within the plots was located by the backpack laser scanning system. Each plot was $30 \mathrm{~m} \times 30 \mathrm{~m}$ in size. The numbers of the reference trees for Plots 1-7 were 29, 54, 54, 18, 20, 21, and 42, respectively. Accordingly, their forest densities were $0.032,0.060,0.060,0.020,0.022,0.023$, and 0.047 trees $/ \mathrm{m}^{2}$, respectively. Within each plot, the location and the diameter at breast height $(\mathrm{DBH})$ of each tree were recorded. 
Table 3. Specifications of the backpack laser scanning system.

\begin{tabular}{cc}
\hline Parameters & Value \\
\hline Weight & $5.8 \mathrm{~kg}$ \\
Working time & $2.5 \mathrm{~h}$ each battery \\
Laser scanning system & Velodyne $16 \mathrm{E}$ \\
Measurement distance range & $100 \mathrm{~m}$ \\
Vertical field angle & $-15-+15 \mathrm{degrees}$ \\
Data accuracy & $<5 \mathrm{~cm}$ \\
\hline
\end{tabular}

\section{Methodology}

To obtain single trees segmented from UAV LiDAR data, our proposed approach includes the following steps: (1) data preprocessing, which first separates ground points from non-ground points and normalizes the UAV LiDAR data according to the produced DTM from the filtered ground points, (2) a voxel-based mean shift method, which voxelizes and roughly segments non-ground points into well-detected and under-segmentation clusters, and (3) an improved normalized cut segmentation method, based on a tree apex detection strategy, which iteratively identifies single trees from the under-segmentation clusters that contain multiple overlapped trees.

\subsection{Data Pre-processing}

In the data preprocessing procedure, to reduce computational complexity, a ground filtering method based on cloth simulation (CSF), developed by Zhang et al. [38], was utilized to classify UAV LiDAR points into ground and non-ground points because it needs a few parameters that are easy to set and adapts to various terrains without tedious parameter setting. The CSF algorithm first turns the terrain upside down and places a cloth on the inverted surface from above, then determines the final shape of the cloth to separate ground from non-ground points by analyzing the interaction between the nodes of the cloth and the corresponding LiDAR points. The CSF algorithm consists of four user-defined parameters: rigidness $\left(F_{R I}\right)$, steep slope fit factor $\left(F_{S T}\right)$, grid resolution $\left(F_{G R}\right)$, and time step $\left(F_{D T}\right)$. The first two parameters are the key parameters, which control the filtering results, and vary with terrain types. The last two parameters are usually fix-valued and universally applicable to all LiDAR datasets. The detailed description of the CSF algorithm can be found in [38]. The ground points were then interpolated into a digital terrain model (DTM) by linear interpolation. Afterward, to reduce the influence of undulating terrain on single-tree recognition and tree height extraction, the non-ground points were normalized according to the produced DTM with a spatial resolution of $0.5 \mathrm{~m}$. Moreover, low-rise shrubs in the forests were removed by a given height threshold $\left(\Gamma_{h}\right)$.

\subsection{Voxel-Based Mean Shift}

The point cloud data, acquired by a UAV LiDAR system, contained a considerable number of points; however, point-wise processing methods are usually time consuming and computationally complex. To reduce data redundancy and improve data processing efficiency, a voxelization strategy was introduced in this study. The non-ground points were segmented into a number of voxels with a given voxel size $\left(V_{s}\right)$. The voxel size depends on the point density of the UAV LiDAR points to be processed. We statistically counted the number of points and determined the center of all the points for each voxel. The voxelization for UAV LiDAR points contributes to improving the computational efficiency and maintaining feature details of objects, which has been widely used for point cloud registration, surface reconstruction, shape recognition, etc.

After voxelization, mean shift, proposed by Ferraz et al. [28], was used to segment single trees roughly with the local maxima based on the density function. All non-ground UAV LiDAR points in the form of voxels can be regarded as a multimodal distribution, where each mode, here defined as a local maximum in both density and height, corresponds to a crown apex [28]. 
The algorithm starts with selecting the highest voxel, $X_{c}=\left(x_{c}, y_{c}, z_{c}\right)$, in the non-ground voxels and obtaining all its neighboring voxels, $X_{i}=\left(x_{i}, y_{i}, z_{i}\right)(i=1,2,3, \ldots, n)$, where $n$ is the number of voxels within a given radius, $R$. Next, we calculated the offset vector by summing the vectors between the voxel and its neighboring voxels. To converge voxels within each crown toward the corresponding crown apex, a 3D space was separated into horizontal and vertical directions. The horizontal kernel was defined for searching local density maxima, and the vertical one for local height maxima [29]. Therefore, the offset vector of voxel $X_{i}$ to voxel $X_{c}$ is defined by:

$$
\mathbf{m}_{h^{s}, h^{r}}\left(X_{c}\right)=\frac{\sum_{i=1}^{n} X_{i} g^{s}\left(\left\|\frac{x_{c}^{s}-x_{i}^{s}}{h^{s}}\right\|^{2}\right) g^{r}\left(\left\|\frac{x_{c}^{r}-x_{i}^{r}}{h^{r}}\right\|^{2}\right)}{\sum_{i=1}^{n} g^{s}\left(\left\|\frac{x_{c}^{s}-x_{i}^{s}}{h^{s}}\right\|^{2}\right) g^{r}\left(\left\|\frac{x_{c}^{r}-x_{i}^{r}}{h^{r}}\right\|^{2}\right)}-X_{c}
$$

where the superscripts $s$ and $r$ refer to the horizontal and vertical directions, respectively. $x_{c}{ }^{s}$ and $x_{c}{ }^{r}$ are the horizontal and vertical vector components of voxel $X_{c}$, respectively. $x_{i}{ }^{s}$ and $x_{i}{ }^{r}$ are the horizontal and vertical vector components of voxel $X_{i}$, respectively. The horizontal and vertical bandwidths $\left(h^{s}, h^{r}\right)$ represent the width and depth of a canopy.

$g^{\mathrm{s}}$ is the horizontal kernel function that follows a Gaussian function:

$$
g^{\mathcal{S}}\left(\left\|\frac{x_{c}^{\mathcal{S}}-x_{i}^{\mathcal{S}}}{h^{\mathcal{S}}}\right\|^{2}\right)=\left\{\begin{array}{ll}
\exp \left(-\frac{1}{2}\left\|\frac{x_{c}^{\mathcal{S}}-x_{i}^{\mathcal{S}}}{h^{s}}\right\|^{2}\right) & \text { if }\left\|\frac{x_{c}^{\mathcal{s}}-x_{i}^{\mathcal{S}}}{h^{s}}\right\| \leq 1 \\
0 & \text { otherwise }
\end{array},\right.
$$

The vertical kernel, $g^{r}$, is specially-designed for assigning a larger weight to the highest voxel:

$$
g^{r}\left(\left\|\frac{x_{c}^{r}-x_{i}^{r}}{h^{r}}\right\|^{2}\right)=\left\{\begin{array}{ll}
1-\left\|1-\operatorname{dist}\left(x_{c}^{r}, x_{i}^{r}\right)\right\|^{2} & \text { if } \operatorname{mask}\left(x_{c}^{r}, x_{i}^{r}\right)=1 \\
0 & \text { otherwise }
\end{array},\right.
$$

where $\operatorname{mask}\left(x_{c}{ }^{r}, x_{i}{ }^{r}\right)$ represents a mask of foreground object; $\operatorname{dist}\left(x_{c}{ }^{r}, x_{i}{ }^{r}\right)$ is the distance between $X_{i}$ and the boundary of the mask. They are defined by,

$$
\begin{gathered}
\operatorname{mask}\left(x_{c}^{r}, x_{i}^{r}\right)= \begin{cases}1 & \text { if } x_{c}^{r}-\frac{h^{r}}{4} \leq x_{i}^{r} \leq x_{c}^{r}+\frac{h^{r}}{2}, \\
0 & \text { otherwise }\end{cases} \\
\operatorname{dist}\left(x_{c}^{r}, x_{i}^{r}\right)= \begin{cases}\min \left(\left\|\frac{\left(x_{c}^{r}-\frac{h^{r}}{4}\right)-x_{i}^{r}}{\frac{3 h^{r}}{8}}\right\|,\left\|\frac{\left(x_{c}^{r}+\frac{h^{r}}{2}\right)-x_{i}^{r}}{\frac{3 h^{r}}{8}}\right\|\right) & \text { if mask }\left(x_{c}^{r}, x_{i}^{r}\right)=1, \\
0 & \text { otherwise }\end{cases}
\end{gathered}
$$

Then, voxel $X_{c}$ moves along the offset vector until it reaches the density and height maxima and labels all voxels visited during this process as the same cluster. The proposed voxel-based mean shift method repeats the above steps until all voxels in the non-ground points are visited and labeled into specific clusters. Afterward, the nearby clusters, whose distances are less than a given distance threshold, $\Gamma_{d}$, are merged together. In the study, to increase the similarity of all voxels belonging to a single tree, the proposed method compresses ( $m$, a multiple of height compression) the point height to improve the clustering results.

With the voxel-based mean shift method, the non-ground voxels were roughly segmented into a set of local clusters. For each cluster, we projected all voxels onto the XOY plane and calculated the diameters in the $\mathrm{x}$ - and $\mathrm{y}$-directions $\left(d_{1}\right.$ and $\left.d_{2}\right)$. According to the ratio of $d_{1}$ to $d_{2}$, the clusters will be classified into two groups: well-detected and under-segmentation clusters. Here, we defined that a well-detected cluster is a single tree with circular-shaped canopy, while an under-segmentation cluster is an irregularly-shaped one containing more than two trees. Thus, if the ratio of $d_{1}$ to $d_{2}$ was close to 1 , the cluster was labeled as well-detected (i.e., a single tree), otherwise the cluster was labeled as under-segmentation. 


\subsection{Improved Normalized Cut Single-Tree Segmentation}

To delineate single trees from the under-segmentation clusters that contain multiple overlapped trees and objects, an improved NCut segmentation algorithm was introduced.

\subsubsection{Normalized Cut}

NCut aims to solve the graph partitioning problem in 2D image segmentation [39]. It works on a weighted connected graph. The idea behind the algorithm is that, by using dynamic programming, a given object is segmented by minimizing the cost for cutting the weighted connected graph into two sub-graphs. The algorithm has been extended to 3D space. The non-empty voxels were used to construct a weighted graph $G=\{V, E\}$ (see Figure 4), where $V$ is a set of graph nodes representing the voxels and $E$ is the set of graph edges that connect the nodes.

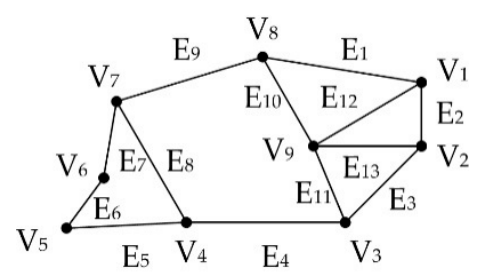

Figure 4. A weighted graph.

Denote weight $\mathbf{w}_{i j}$ as the similarity between a pair of nodes $\{i, j\} \in V$. It is computed as follows:

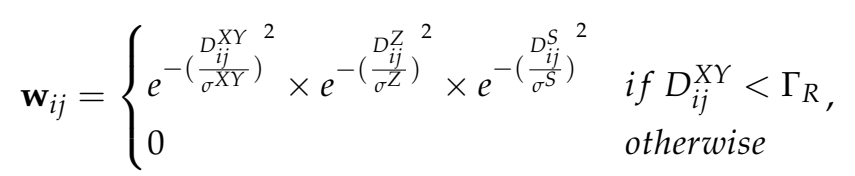

where $D_{i j}{ }^{X Y}, D_{i j}{ }^{Z}$, and $D_{i j}{ }^{S}$ represent the horizontal, vertical, and shortest distances, respectively, between nodes $i$ and j. $\sigma^{X Y}, \sigma^{X Y}, \sigma^{Z}, \sigma^{Z}$, and $\sigma^{S}$ are coefficients, set to be 0.05-times the maximum of $D_{i j}{ }^{X Y}, D_{i j}{ }^{Z}$, and $D_{i j}{ }^{S}$, for controlling the sensitivity of the impact factors, respectively. $\Gamma_{R}$ represents the maximum horizontal distance threshold between nodes. $\mathbf{w}_{i j}=0$, if the horizontal distance between nodes $\{i, j\}$ exceed the threshold, $\Gamma_{R}$. NCut aims to divide the graph $G$ into two disjoint voxel groups $A$ and $B$ by maximizing the similarity within each voxel group and minimizing the similarity between two voxel groups $A$ and $B$. The corresponding cost function is as follows:

$$
\operatorname{NCut}(A, B)=\frac{\operatorname{Cut}(A, B)}{A \operatorname{ssoc}(A, V)}+\frac{\operatorname{Cut}(B, A)}{A \operatorname{ssoc}(B, V)},
$$

where $\operatorname{Cut}(A, B)=\sum_{i \in A, j \in B} w_{i j}$ is the sum of weights between voxel groups $A$ and $B$. $\operatorname{Assoc}(A, V)=\sum_{i \in A, j \in V} w_{i j}$ and $A \operatorname{ssoc}(B, V)=\sum_{i \in B, j \in V} w_{i j}$ represent the sums of weights of all edges ending in the voxel groups $A$ and $B$, respectively. To segment voxel groups $A$ and $B$ exactly, $\operatorname{NCut}(A, B)$ is minimized and solved by the corresponding generalized eigenvalue equation:

$$
(\mathbf{D}-\mathbf{W}) y=\lambda \mathbf{D} y,
$$

where $\mathbf{W}$ is an $n \times n$ weighting matrix representing the weights between all nodes of graph $G$. $\mathbf{D}$ is an $n \times n$ diagonal matrix, and

$\mathbf{D}(i, i)=\sum_{j} w i j . \quad \lambda$ is an eigenvalue, and $y$ represents the eigenvector corresponding to the generalized eigenvalue. The minimum solution, $y_{1}$, to Equation (8) corresponds to the second smallest eigenvalue, $\lambda_{1}$ [23]. 


\subsubsection{Improved NCut}

The traditional NCut algorithm has difficulty in accurately locating tree stems in airborne/UAV LiDAR data due to the lack of understory information in severely-overlapped forests. NCut requires the specific number of single trees in point cloud data to be processed. Therefore, in the study, to segment single trees from the under-segmentation clusters containing multiple overlapped objects, we improved the NCut algorithm by adopting a tree apex detection strategy, which is capable of automatically determining the number of single trees in the clusters. The tree apex detection strategy starts with profile generation, which sections the cluster into a set of profiles both in the $x$ - and $y$-direction with a user-defined profile size, $\Gamma_{s}$. The profiles generated both in the $x$ - and y-direction help reduce the occlusions. For each profile in the $x$ - and y-direction, respectively, we calculate the maximum height for generating shape curves by cubic spline data interpolation. Figure 5 shows two clusters and their corresponding shape curves. Figure $5 \mathrm{a}$,d show two clusters in the form of 3D points, Figure $5 b$,e their corresponding shape curves in the $x$-direction and Figure $5 c, f$ the shape curves in the $y$-direction. The peaks of the generated shape curves both in the $\mathrm{x}$ - and $\mathrm{y}$-direction are found to determine tree apices, which means the number of peaks in the shape curves determines the number of potential single trees to be segmented in the under-segmentation cluster.

With the determined number of potential tree apices, the NCut iteratively segments the cluster into the specified number of single trees. Figure 6a shows an example of an under-segmentation cluster with multiple overlapped trees. As seen in Figure 6b, the cluster is further segmented into six potential single trees.

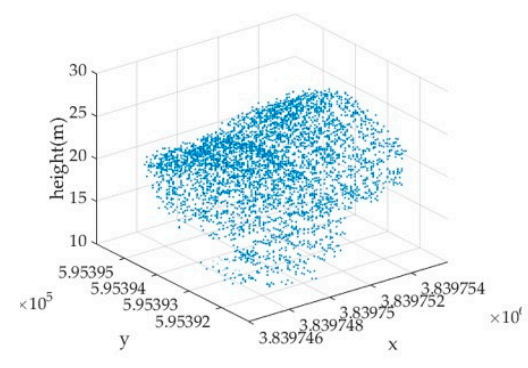

(a)

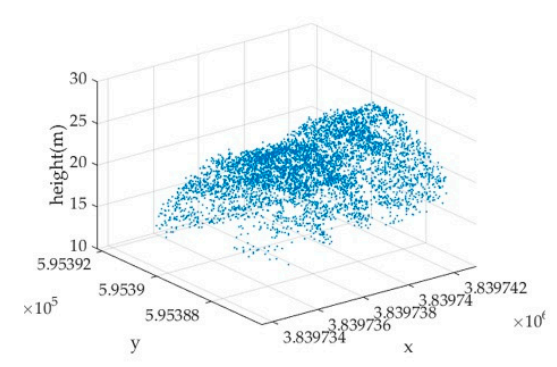

(d)

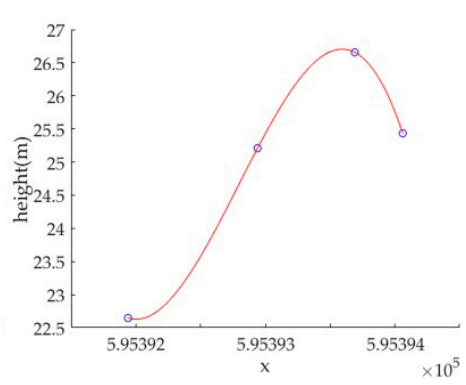

(b)

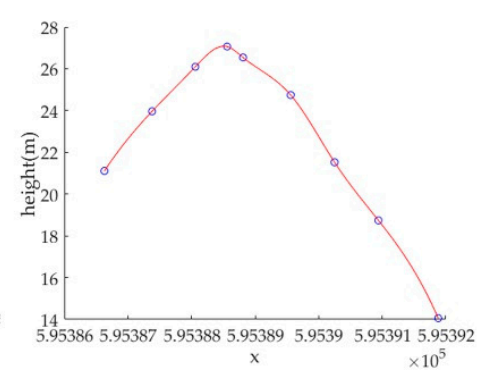

(e)

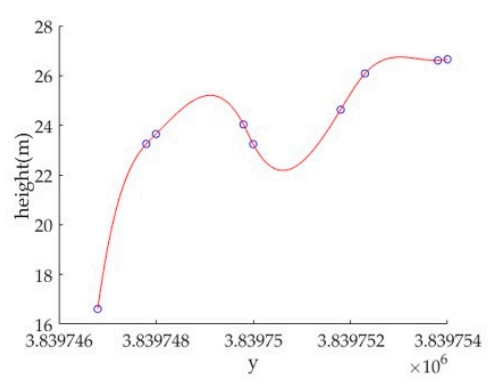

(c)

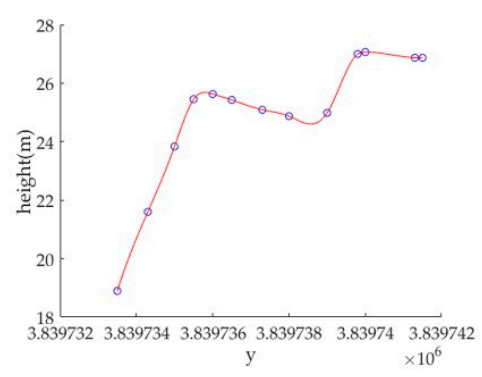

(f)

Figure 5. Two-cluster examples and their corresponding shape curves both in the $x$ - and $y$-direction: $(\mathbf{a}, \mathbf{d})$ two-cluster samples, $(\mathbf{b}, \mathbf{e})$ their shape curves in the $\mathbf{x}$-direction, and $(\mathbf{c}, \mathbf{f})$ their shape curves in the y-direction.

\section{Experimental Results and Discussion}

The proposed single-tree segmentation method was performed using Microsoft Visual Studio 2013 (programed using $\mathrm{C}++$ ) and MATLAB 2016a and tested on an HP Z820 eight-core-16-thread workstation. To evaluate the proposed single-tree segmentation method qualitatively and quantitatively, we conducted a set of experiments on seven reference plots. In this study, we segmented 
single trees based on tree apices, which might lead to an identification error between the location of the segmented single tree and the reference data. If the identification error was within a certain range or the majority of points (over $80 \%$ ) belonging to the segmented single tree were correctly identified, we considered that the single tree was correctly identified.

Before we investigated the applicability and feasibility of the proposed single-tree segmentation method, several parameters were empirically selected in advance, according to prior acknowledge of the UAV LiDAR data and the test site. As seen in Table 4, in the data preprocessing, five parameters $-F_{R I}, F_{S T}, F_{G R}, F_{D T}$, and $\Gamma_{h}$-were used. $F_{R I}$ represented the terrain type and was set to three according to the flat terrain. $F_{S T}$ decided whether the post-processing of steep slopes was required. $F_{G R}$ and $F_{D T}$ were respectively set to 0.5 and 0.65 , which were adapted to most of the scenes. $F_{G R}$ represented the horizontal distance between two neighboring points, and $F_{D T}$ controlled the displacement of points from gravity during each iteration. Low-rise shrub height threshold, $\Gamma_{h}$, was set to $5.0 \mathrm{~m}$ according to the tree height in the test site. In the voxel-based mean shift algorithm, six parameters- $V_{s}, m, R, \Gamma_{d}, h_{s}$, and $h_{r}$-were used to obtain a set of clusters. The voxel size, $V_{s}$, was set according to the point density. To increase the similarity in vertical distance, $m$ was set to four. The search radius, $R$, and the minimum distance between clusters, $\Gamma_{d}$, were both set to $2.0 \mathrm{~m}$ according to the average width of canopies in the study. $h_{s}$ and $h_{r}$ were two bandwidths of the horizontal and vertical kernels, which represented the range where the local density and local maxima existed. $h_{s}$ and $h_{r}$ were set to $1.5 \mathrm{~m}$ and $5.0 \mathrm{~m}$, respectively, according to the average width and depth of the tree canopies in the test site. In the improved NCut-based single-tree segmentation stage, $\Gamma_{s}$, the profile size in the $\mathrm{x}$ - and $\mathrm{y}$-direction was set to $0.5 \mathrm{~m}$ based on the defined voxel size. The maximum horizontal distance threshold between the cluster nodes, $\Gamma_{R}$, was empirically set to $4.5 \mathrm{~m}$ according to the average width of tree canopies.

Table 4. Parameters and configurations in the proposed method.

\begin{tabular}{cccc}
\hline Stage & Parameter & Definition & Value \\
\hline \multirow{3}{*}{ Data preprocessing } & $\Gamma_{h}$ & Low-rise shrubs height threshold & $5.0 \mathrm{~m}$ \\
& $F_{R I}$ & Terrain types & 3 \\
& $F_{S T}$ & Steep slope fit factor & False \\
& $F_{G R}$ & Grid resolution & 0.5 \\
& $F_{D T}$ & Time step & 0.65 \\
\hline \multirow{2}{*}{ Voxel-based mean shift } & $V_{S}$ & Voxel size & $0.2 \mathrm{~m}$ \\
& $m$ & Height compression & 4 \\
& $R$ & Search radius & $2.0 \mathrm{~m}$ \\
& $h^{s}$ & Horizontal bandwidth & $1.5 \mathrm{~m}$ \\
& $h^{r}$ & Vertical bandwidth & $5.0 \mathrm{~m}$ \\
Improved NCut segmentation & $\Gamma_{d}$ & Minimum distance between clusters & $2.0 \mathrm{~m}$ \\
\hline
\end{tabular}

The accuracy of the proposed method was evaluated by the following three measurements: correctness $\left(E_{c o r}\right)$, completeness $\left(E_{c p t}\right)$, and $F$-score $\left(E_{f}\right)$. $E_{c o r}$ indicates what percentage of the segmented single trees were valid, whereas $E_{c p t}$ describes how complete the detected single trees were. $E_{c o r}$ is defined as $E_{c o r}=C_{p} / E_{p}$, and $E_{c p t}$ is expressed as $E_{c p t}=C_{p} / R f$, where $C_{p}$ denotes the number of real single trees, $R f$ is the number of single trees in the reference data, and $E_{p}$ represents the number of single trees segmented by our method. $E_{f}$ evaluates the overall accuracy, which is defined as:

$$
E_{f}=2\left(E_{c o r} \times E c p t\right) /\left(E_{c o r}+E c p t\right)
$$




\subsection{Overall Performance}

To evaluate the performance of our proposed single-tree segmentation method, we applied it to the UAV LiDAR data set. Two tree species, Metasequoia and Poplar, were tested. The parameters involved in this study were set according to Table 4 . The UAV LiDAR data in the test site were first preprocessed to obtain the normalized non-ground points. The ground points were compared with the reference DTM, which was manually processed by means of a Terrasolid@ software (http:/ / www.terrasolid. com/home.php). The root mean squared error (RMSE) was $0.23 \mathrm{~m}$, referring to the evaluation method in [40]. Then, with the voxel-based mean shift algorithm, the non-ground UAV points were compressed and roughly segmented into a set of clusters (See Figure 7). As shown in Figure 7, visual inspection demonstrated that the voxel-based mean shift algorithm roughly classified the non-ground UAV voxels into well-detected and under-segmentation clusters. According to the calculated mean shift vectors that always pointed to the local density and height maxima in the non-ground UAV voxels, the clusters with distinct tree apices were classified as well detected. The well-detected clusters were then directly labeled as single trees. However, the clusters without distinct tree apex structures, labeled as under-segmentation, needed to be further processed. An under-segmentation cluster contains multiple, overlapped tree canopies or small trees. As seen in Figure 7, the segmentation results of Metasequoia outperformed those of Poplar. This is because Poplar has complex tree structures with staggered stems and irregularly-shaped canopies. On the contrary, over-segmentation phenomena existed for Poplar, as seen in Figure 7d-g.

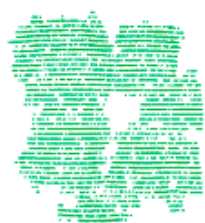

(a)

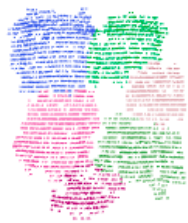

(b)

Figure 6. An example of the improved NCut segmentation algorithm: (a) a cluster containing multiple overlapped trees and (b) improved NCut segmentation results (different colors representing different single trees).

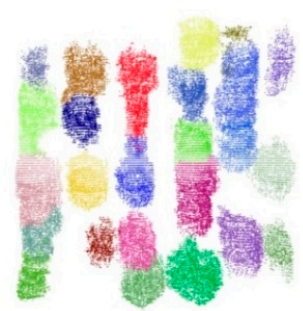

(a)

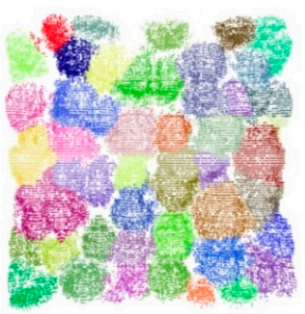

(b)

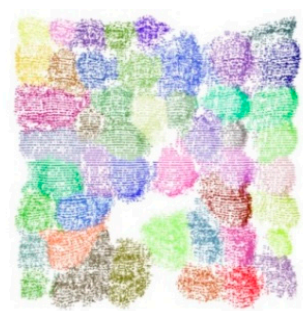

(c)

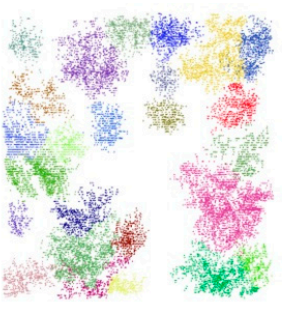

(d)

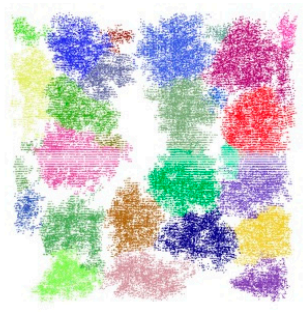

(e)

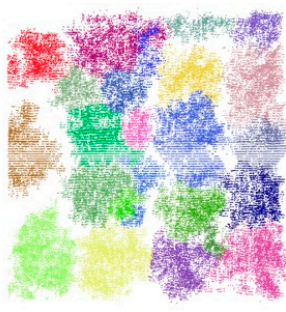

(f)

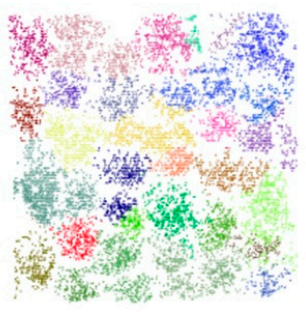

(g)

Figure 7. Results of the voxel-based mean shift method for seven plots: (a) Plot 1, (b) Plot 2, (c) Plot 3, (d) Plot 4, (e) Plot 5, (f) Plot 6, and (g) Plot 7. 
To further detect single trees from the under-segmentation clusters, the improved NCut segmentation method was performed. For each under-segmentation cluster, tree apices, based on the tree shape curves generated by the vertical profile information both in the $\mathrm{x}$ - and $\mathrm{y}$-directions, were found and input to the NCut method to obtain single trees by iteratively segmenting the cluster. Table 5 shows the single-tree segmentation results by our proposed approach. As seen in Table 5, most single trees have been correctly segmented with an average correctness of 0.90 , completeness of 0.88 , and $E_{f}$ value of 0.89 , respectively. For Metasequoia (Plots 1, 2, and 3), the $E_{c o r}$ values were greater than 0.96, the $E_{c p t}$ values were higher than 0.88 , and the $E_{f}$ values ranged from 0.93-0.94. The quantitative results indicate that the proposed method was greatly suitable for detecting the deciduous Metasequoia with the cone-shaped canopies, sparse branches, and leaves. For Poplar (Plots 4, 5, 6, and 7) with the irregularly-shaped canopies and staggered-like stems, the values of $E_{c o r}, E_{c p t}$, and $E_{f}$ ranged from $0.81-0.9$, slightly lower than those of the Metasequoia trees. Overall, the experiments indicate that our approach was robust to different tree species.

Moreover, according to the forest density statistics in Table 5, we found that for Metasequoia, slight improvements of the $E_{c o r}$ values were achieved for Plots 2 and 3 with the highest forest densities of 0.060 trees $/ \mathrm{m}^{2}$ when compared to Plot 1 with a forest density of 0.032 trees $/ \mathrm{m}^{2}$. On the contrary, the $E_{f}$ values of Plots 2 and 3 were slightly lower than that of Plot 1 . For Poplar, Plots 4-6 with little differences in forest density achieved $E_{f}$ values ranging from $0.83-0.87$. Compared to Plot 4 with the lowest forest density of 0.020 , an improvement of the $E_{c o r}, E_{c p t}$, and $E_{f}$ values of Plot 7 was achieved by $0.05,0.02$, and 0.03 , respectively. This indicates that our approach was capable of processing dense forests with overlapped canopies.

Table 5. Single tree segmentation results by our approach.

\begin{tabular}{ccccc}
\hline & Forest Density $\left(\right.$ trees $\left./ \mathbf{m}^{\mathbf{2}}\right)$ & $\boldsymbol{E}_{\text {cor }} \mathbf{( \% )}$ & $\boldsymbol{E}_{\boldsymbol{c p t}} \mathbf{( \% )}$ & $\boldsymbol{E}_{\boldsymbol{f}}$ \\
\hline Plot 1 & 0.032 & 0.96 & 0.93 & 0.94 \\
Plot 2 & 0.060 & 0.98 & 0.93 & 0.93 \\
Plot 3 & 0.060 & 0.98 & 0.88 & 0.93 \\
Plot 4 & 0.020 & 0.84 & 0.88 & 0.86 \\
Plot 5 & 0.022 & 0.89 & 0.85 & 0.87 \\
Plot 6 & 0.023 & 0.81 & 0.85 & 0.83 \\
Plot 7 & 0.047 & 0.89 & 0.9 & 0.89 \\
Average accuracy & & 0.90 & 0.88 & 0.89 \\
\hline
\end{tabular}

${ }^{1} E_{\text {cor }}$ indicates what percentage of the segmented single trees are valid; $E_{c p t}$ describes how complete the detected single trees are; and $E_{f}$ evaluates the overall accuracy.

\subsection{Comparative Tests}

A comparative test was carried out to compare our method with the marker-controlled watershed segmentation [12]. The method was performed using LiDAR360 produced by Green Valley International (https:/ /www.lidar360.com/archives/5135.html), and Figure 8 was drawn by Arcgis10.2 produced by the Environmental Systems Research Institute (ESRI). Figure 8 and Table 6, qualitatively and quantitatively, show the watershed segmentation results for the seven plots.

As seen in Figure 8a-c, for the cone-shaped, orderly-arranged Metasequoia trees, the watershed segmentation method performed very well. Accordingly, as seen in Table 6, the values of $E_{f}$ were 0.98 , 0.92 , and 0.92 , respectively. A few trees were over-segmented or missed. The average $E_{f}$ value of the marker-controlled watershed segmentation algorithm was slightly lower than that of our approach. This is because the watershed segmentation algorithm was sensitive to noise, which leads to staggered stems probably being misclassified into tree seed points. Moreover, the watershed segmentation algorithm detects single trees from the 2D CHM data interpolated from 3D points, which means data interpolation might decrease the accuracies of canopy segmentation and cause the unreliability of delineating crown diameters. For the Poplar trees (Plots 4, 5, 6, and 7), Figure 9a,b shows a close segmentation example of Plot 5 by the watershed segmentation and our proposed approach. Green 
dots and black circles represent tree locations and canopy radii derived from watershed segmentation, respectively, and black crosses represent reference tree locations. As seen in Figure 9a, the single trees detected by local maxima in CHM data were over-segmented, which was mainly caused by staggered stems, and tree canopies were still overlapped with each other, indicating that the segmentation results were unsatisfactory. Quantitatively, as seen in Table 6, the $E_{c o r}$ values greatly ranged from 0.75-0.91, the $E_{c p t}$ values changed from 0.83-0.90, and the values of $E_{f}$ for the four Poplar plots only attained $0.79,0.80,0.85$, and 0.84 , respectively. This is because the local maxima detection in $2 \mathrm{D}$ CHM data, which only contain height difference information, is unreliable for dealing with the trees with irregularly-shaped canopies and staggered-like branches, and thus, many Poplar trees were over-segmented and missed.

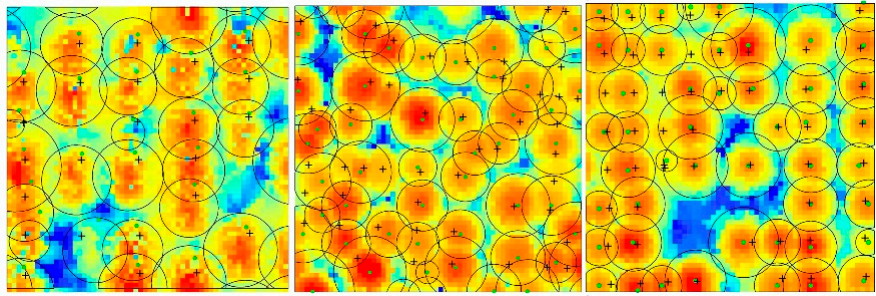

(a)

(b)

(c)

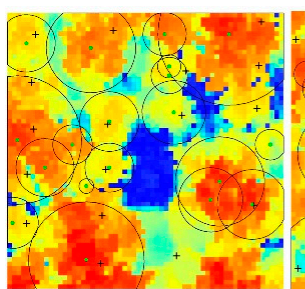

(d)

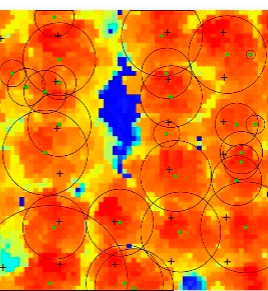

(e)

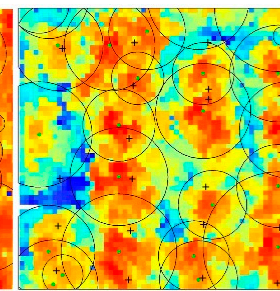

(f)

(g)

Figure 8. The segmentation results of marker-controlled watershed segmentation for seven plots: (a) Plot 1, (b) Plot 2, (c) Plot 3, (d) Plot 4, (e) Plot 5, (f) Plot 6, and (g) Plot 7.

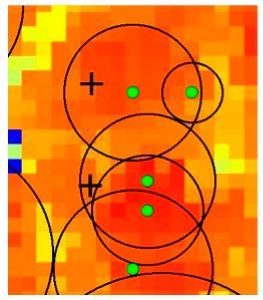

(a)

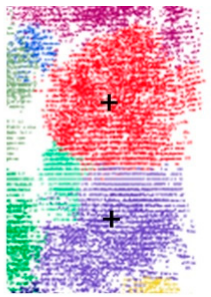

(b)

Figure 9. An example of segmentation results for Plot 5: (a) the watershed segmentation and (b) our proposed approach.

Table 6. Single-tree segmentation results by the marker-controlled watershed segmentation method.

\begin{tabular}{ccccc}
\hline & Forest Density $\left(\right.$ trees $\left./ \mathbf{m}^{\mathbf{2}}\right)$ & $E_{\text {cor }} \mathbf{( \% )}$ & $E_{\text {cpt }} \mathbf{( \% )}$ & $E_{f}$ \\
\hline Plot 1 & 0.032 & 0.96 & 1.00 & 0.98 \\
Plot 2 & 0.060 & 0.92 & 0.92 & 0.92 \\
Plot 3 & 0.060 & 0.94 & 0.91 & 0.92 \\
Plot 4 & 0.020 & 0.75 & 0.83 & 0.79 \\
Plot 5 & 0.022 & 0.75 & 0.90 & 0.80 \\
Plot 6 & 0.023 & 0.90 & 0.82 & 0.85 \\
Plot 7 & 0.047 & 0.91 & 0.81 & 0.84 \\
Average accuracy & & 0.87 & 0.88 & 0.87 \\
\hline
\end{tabular}

${ }^{1} E_{c o r}$ indicates what percentage of the segmented single trees are valid; $E_{c p t}$ describes how complete the detected single trees are; and $E_{f}$ evaluates the overall accuracy. 
For the Metasequoia test plots (i.e., Plots 1, 2, and 3), our proposed approach and the marker-controlled watershed segmentation method achieved similar single-tree delineation performances, both satisfactorily at delineating single trees from the cone-shaped, orderly-arranged Metasequoia forest. Moreover, for Poplar trees with irregularly-shaped canopies and staggered-like branches, the 2D CHM-based watershed segmentation method achieved unreliable results due to the loss of some tree-related information in the data interpolation. Compared to the marker-controlled watershed segmentation method, our approach was robust to forests with different tree species and forest densities by finding both local density and height maxima directly from 3D point clouds.

\section{Conclusions}

This paper proposed a hierarchical single-tree segmentation approach using UAV LiDAR data, which consists of data preprocessing, voxel-based mean-shift clustering, and delineation of single trees by the improved NCut segmentation with a tree apex detection strategy.

The test data acquired by a Velodyne 16E UAV LiDAR system were used in this paper to assess our single-tree segmentation method. We selected seven plots containing two tree species with different forest densities. The experimental results demonstrated that our approach was capable of delineating single trees with an average correctness of 0.90 , an average completeness of 0.88 , and an average overall accuracy of 0.89 , respectively. Our approach is robust and highly efficient for segmenting single trees from UAV LiDAR data with high point cloud density since: (1) voxelization facilitates improving the efficiency of data processing; (2) the full use of the 3D spatial structure of the point cloud rather than the interpolated 2D CHM data, which only contain height difference information, in voxel-based mean shift improves tree apex location accuracy due to less loss of tree information; (3) a profile shape curve strategy based on vertical profile information contributes to locating tree apices and solving the problem that NCut is incapable of detecting single trees from overlapped canopies due to the lack of understory information in severely-overlapped forests.

Author Contributions: W.Y. conceived of and designed the experiments and performed the experiments; H.G. and Y.Y. analyzed the data; H.G., L.C., and J.L. revised the paper. L.C. and S.G. did the field works and preprocessed the data.

Funding: This work was supported in part by the National Natural Science Foundation of China under Grant 41671454 and Grant 61603146, in part by the Natural Science Foundation of Jiangsu Province under Grant BK20160427, in part by the Natural Science Research in Colleges and Universities of Jiangsu Province under Grant 16KJB520006, and in part by the Science and Technology Project of Huaian City under Grant HAG201602. Contact the corresponding author via guanhy.nj@nuist.edu.cn for more information about the software tools used in this study.

Conflicts of Interest: The authors declare no conflict of interest.

\section{Abbreviations}

The following abbreviations are used in this manuscript:

$\begin{array}{ll}\text { UAV } & \text { Unmanned Aerial Vehicle } \\ \text { NCut } & \text { Normalized cut segmentation } \\ \text { LiDAR } & \text { Light detection and ranging } \\ \text { 2D } & \text { Two-dimensional } \\ \text { 3D } & \text { Three-dimensional } \\ \text { CHM } & \text { Canopy height model } \\ \text { CSF } & \text { Cloth simulation filtering } \\ \text { DTM } & \text { Digital terrain model } \\ \text { DBH } & \text { Diameter at breast height } \\ \text { VLP16 } & \text { Velodyne 16E laser scanning system } \\ \text { GPS } & \text { Global positioning system }\end{array}$




\section{References}

1. Hyyppä, J.; Yu, X.; Hyyppä, H.; Vastaranta, M.; Holopainen, M.; Kukko, A.; Kaartinen, H.; Jaakkola, A.; Vaaja, M.; Koskinen, J.; et al. Advances in Forest Inventory Using Airborne Laser Scanning. Remote Sens. 2012, 4, 1190-1207. [CrossRef]

2. Matasci, G.; Hermosilla, T.; Wulder, M.A.; White, J.C.; Coops, N.C.; Hobart, G.W.; Zald, H.S.J. Large-area mapping of Canadian boreal forest cover, height, biomass and other structural attributes using Landsat composites and lidar plots. Remote Sens. Environ. 2018, 209, 90-106. [CrossRef]

3. Allouis, T.; Durrieu, S.; Vega, C.; Couteron, P. Stem Volume and Above-Ground Biomass Estimation of Individual Pine Trees from LiDAR Data: Contribution of Full-Waveform Signals. IEEE J. Sel. Top. Appl. Earth Obs. Remote Sens. 2013, 6, 924-934. [CrossRef]

4. Matsuki, T.; Yokoya, N.; Iwasaki, A. Hyperspectral Tree Species Classification of Japanese Complex Mixed Forest with the Aid of Lidar Data. IEEE J. Sel. Top. Appl. Earth Obs. Remote Sens. 2017, 8, 2177-2187. [CrossRef]

5. Wu, J.; Yao, W.; Polewski, P. Mapping Individual Tree Species and Vitality along Urban Road Corridors with LiDAR and Imaging Sensors: Point Density versus View Perspective. Remote Sens. 2018, 10, 1403. [CrossRef]

6. Barnes, C.; Balzter, H.; Barrett, K.; Eddy, J.; Milner, S.; Suárez, J. Individual Tree Crown Delineation from Airborne Laser Scanning for Diseased Larch Forest Stands. Remote Sens. 2017, 9, 231. [CrossRef]

7. Nyström, M.; Holmgren, J.; Fransson, J.E.S.; Olsson, H. Detection of windthrown trees using airborne laser scanning. Int. J. Appl. Earth Obs. Geoinf. 2014, 30, 21-29. [CrossRef]

8. Paris, C.; Valduga, D.; Bruzzone, L. A Hierarchical Approach to Three-Dimensional Segmentation of LiDAR Data at Single-Tree Level in a Multilayered Forest. IEEE Trans. Geosci. Remote Sens. 2016, 54, 4190-4203. [CrossRef]

9. Polewski, P.; Yao, W.; Heurich, M.; Krzystek, P.; Stilla, U. Detection of fallen trees in ALS point clouds using a Normalized Cut approach trained by simulation. ISPRS J. Photogramm. Remote Sens. 2015, 105, $252-271$. [CrossRef]

10. Zhang, C.; Zhou, Y.; Qiu, F. Individual Tree Segmentation from LiDAR Point Clouds for Urban Forest Inventory. Remote Sens. 2015, 7, 7892-7913. [CrossRef]

11. Yu, X.; Hyyppä, J.; Litkey, P.; Kaartinen, H.; Vastaranta, M.; Holopainen, M. Single-Sensor Solution to Tree Species Classification Using Multispectral Airborne Laser Scanning. Remote Sens. 2017, 9, 108. [CrossRef]

12. Latifi, H.; Fassnacht, F.E.; Müller, J.; Tharani, A.; Dech, S.; Heurich, M. Forest inventories by LiDAR data: A comparison of single tree segmentation and metric-based methods for inventories of a heterogeneous temperate forest. Int. J. Appl. Earth Obs. Geoinf. 2015, 42, 162-174. [CrossRef]

13. Vega, C.; Hamrouni, A.; El Mokhtari, S.; Morel, J.; Bock, J.; Renaud, J.P.; Bouvier, M.; Durrieu, S. PTrees: A point-based approach to forest tree extraction from lidar data. Int. J. Appl. Earth Obs. Geoinf. 2014, 33, 98-108. [CrossRef]

14. Koc-San, D.; Selim, S.; Aslan, N.; San, B.T. Automatic citrus tree extraction from UAV images and digital surface models using circular Hough transform. Comput. Electron. Agric. 2018, 150, 289-301. [CrossRef]

15. Wallace, L.; Lucieer, A.; Watson, C.S. Evaluating Tree Detection and Segmentation Routines on Very High Resolution UAV LiDAR Data. IEEE Trans. Geosci. Remote Sens. 2014, 52, 7619-7628. [CrossRef]

16. Tian, J.; Wang, L.; Li, X.; Gong, H.; Shi, C.; Zhong, R.; Liu, X. Comparison of UAV and WorldView-2 imagery for mapping leaf area index of mangrove forest. Int. J. Appl. Earth Obs. Geoinf. 2017, 61, 22-31. [CrossRef]

17. Sankey, T.; Donager, J.; McVay, J.; Sankey, J.B. UAV lidar and hyperspectral fusion for forest monitoring in the southwestern USA. Remote Sens. Environ. 2017, 195, 30-43. [CrossRef]

18. Kaartinen, H.; Hyyppä, J.; Yu, X.; Vastaranta, M.; Hyyppä, H.; Kukko, A.; Holopainen, M.; Heipke, C.; Hirschmugl, M.; Morsdorf, F.; et al. An International Comparison of Individual Tree Detection and Extraction Using Airborne Laser Scanning. Remote Sens. 2012, 4, 950-974. [CrossRef]

19. Leckie, D.; Gougeon, F.; Hill, D.; Quinn, R.; Armstrong, L.; Shreenan, R. Combined high-density lidar and multispectral imagery for individual tree crown analysis. Can. J. Remote Sens. 2003, 29, 633-649. [CrossRef]

20. Chen, Q.; Baldocchi, D.; Gong, P.; Kelly, M. Isolating Individual Trees in a Savanna Woodland Using Small Footprint Lidar Data. Photogramm. Eng. Remote Sens. 2006, 72, 923-932. [CrossRef]

21. Lee, H.; Slatton, K.C.; Roth, B.E.; Cropper, W.P. Adaptive clustering of airborne LiDAR data to segment individual tree crowns in managed pine forests. Int. J. Remote Sens. 2010, 31, 117-139. [CrossRef] 
22. Li, W.; Guo, Q.; Jakubowski, M.K.; Kelly, M. A New Method for Segmenting Individual Trees from the Lidar Point Cloud. Photogramm. Eng. Remote Sens. 2012, 78, 75-84. [CrossRef]

23. Vauhkonen, J.; Ene, L.; Gupta, S.; Heinzel, J.; Holmgren, J.; Pitkanen, J.; Solberg, S.; Wang, Y.; Weinacker, H.; Hauglin, K.M.; et al. Comparative testing of single-tree detection algorithms under different types of forest. Forestry 2011, 85, 27-40. [CrossRef]

24. Jakubowski, M.K.; Li, W.; Guo, Q.; Kelly, M. Delineating Individual Trees from Lidar Data: A Comparison of Vector- and Raster-based Segmentation Approaches. Remote Sens. 2013, 5, 4163-4186. [CrossRef]

25. Lu, X.; Guo, Q.; Li, W.; Flanagan, J. A bottom-up approach to segment individual deciduous trees using leaf-off lidar point cloud data. ISPRS J. Photogramm. Remote Sens. 2014, 94, 1-12. [CrossRef]

26. Morsdorf, F.; Meier, E.; Kötz, B.; Itten, K.I.; Dobbertin, M.; Allgöwer, B. LIDAR-based geometric reconstruction of boreal type forest stands at single tree level for forest and wildland fire management. Remote Sens. Environ. 2004, 92, 353-362. [CrossRef]

27. Gupta, S.; Weinacker, H.; Koch, B. Comparative Analysis of Clustering-Based Approaches for 3-D Single Tree Detection Using Airborne Fullwave Lidar Data. Remote Sens. 2010, 2, 968-989. [CrossRef]

28. Ferraz, A.; Bretar, F.; Jacquemoud, S.; Gonçalves, G.; Pereira, L.; Tomé, M.; Soares, P. 3-D mapping of a multi-layer ed Mediterranean forest using ALS data. Remote Sens. Environ. 2012, 121, 210-223. [CrossRef]

29. Dai, W.; Yang, B.; Dong, Z.; Shaker, A. A new method for 3D individual tree extraction using multispectral airborne LiDAR point clouds. ISPRS J. Photogramm. Remote Sens. 2018, 144, 400-411. [CrossRef]

30. Reitberger, J.; Schnörr, C.; Krzystek, P.; Stilla, U. 3D segmentation of single trees exploiting full waveform LIDAR data. ISPRS J. Photogramm. Remote Sens. 2009, 64, 561-574. [CrossRef]

31. Zhong, L.; Cheng, L.; Xu, H.; Wu, Y.; Chen, Y.; Li, M. Segmentation of Individual Trees From TLS and MLS Data. IEEE J. Sel. Top. Appl. Earth Obs. Remote Sens. 2017, 10, 774-787. [CrossRef]

32. Yu, Y.; Li, J.; Guan, H.; Wang, C. Automated Extraction of Urban Road Facilities Using Mobile Laser Scanning Data. IEEE Trans. Intell. Transp. Syst. 2015, 16, 2167-2181. [CrossRef]

33. Yao, W.; Krzystek, P.; Heurich, M. Tree species classification and estimation of stem volume and DBH based on single tree extraction by exploiting airborne full-waveform LiDAR data. Remote Sens. Environ. 2012, 123, 368-380. [CrossRef]

34. Lin, Y.; Hyyppa, J.; Jaakkola, A. Mini-UAV-Borne LIDAR for Fine-Scale Mapping. IEEE Geosci. Remote Sens. Lett. 2011, 8, 426-430. [CrossRef]

35. Liu, K.; Shen, X.; Cao, L.; Wang, G.; Cao, F. Estimating forest structural attributes using UAV-LiDAR data in Ginkgo plantations. ISPRS J. Photogramm. Remote Sens. 2018, 146, 465-482. [CrossRef]

36. Harwin, S.; Lucieer, A. Assessing the Accuracy of Georeferenced Point Clouds Produced via Multi-View Stereopsis from Unmanned Aerial Vehicle (UAV) Imagery. Remote Sens. 2012, 4, 1573-1599. [CrossRef]

37. Wallace, L.; Lucieer, A.; Watson, C.; Turner, D. Development of a UAV-LiDAR System with Application to Forest Inventory. Remote Sens. 2012, 4, 1519-1543. [CrossRef]

38. Zhang, W.; Qi, J.; Wan, P.; Wang, H.; Xie, D.; Wang, X.; Yan, G. An Easy-to-Use Airborne LiDAR Data Filtering Method Based on Cloth Simulation. Remote Sens. 2016, 8, 501. [CrossRef]

39. Jianbo, S.; Malik, J. Normalized cuts and image segmentation. IEEE Trans. Pattern Anal. Mach. Intell. 2000, 22, 888-905. [CrossRef]

40. Balenović, I.; Gašparović, M.; Milas, A.S.; Berta, A.; Seletković, A. Accuracy Assessment of Digital Terrain Models of Lowland Pedunculate Oak Forests Derived from Airborne Laser Scanning and Photogrammetry. Croat. J. For. Eng. 2018, 39, 117-128.

(c) 2018 by the authors. Licensee MDPI, Basel, Switzerland. This article is an open access article distributed under the terms and conditions of the Creative Commons Attribution (CC BY) license (http://creativecommons.org/licenses/by/4.0/). 